\title{
Proposed Technique of Analysis for Absorber/Transmitter Frequency Selective Surfaces
}

\author{
Eliel P. dos Santos ${ }^{1} \mathbb{D}$, Antonio L. P. S. Campos $^{2}{ }^{\mathbb{D}}$, Alfredo Gomes Neto ${ }^{3}$, Maurício W. B. Silva ${ }^{4}$ \\ ${ }^{1}$ Federal University of Tocantins, Palmas, TO, Brazil, CEP: 77001090, email: elielpoggi@gmail.com \\ ${ }^{2}$ Federal University of Rio Grande do Norte, Natal, RN, Brazil, alpscampos@gmail.com \\ ${ }^{3}$ Federal Institute of Paraiba, João Pessoa, PB, Brazil, alfredogomesjpa@gmail.com \\ ${ }^{4}$ Federal University Fluminense, Niteroi, RJ, Brazil, mauricio.weber@gmail.com
}

\begin{abstract}
In this paper, we propose a simple technique to analyze absorber/transmitter frequency selective surfaces (FSS). The structure is formed by two FSS, one resistive and one conductive. Both FSS are printed on an FR4 dielectric substrate. The square loop and crossed dipole are the geometries considered in this paper. The technique combines two simple approaches, the equivalent circuit method, and the scattering matrix method. Two prototypes were built for validation purposes. Simulated and measured results are in good agreement.
\end{abstract}

Index Terms - Absorber/Transmitter FSS, Simple Numerical Technique.

\section{INTRODUCTION}

With the rapid advances of wireless communications, electromagnetic wave absorbers are becoming increasingly important for space field applications such as anechoic chambers, radar systems, and military applications [1]. Recent researches on electromagnetic wave absorbers have been developed in order to improve indoor environments due to the proliferation of wireless local area network (WLAN) systems [2], [3].

In the middle of World War II, aircraft were detected through the radar cross-section (RCS), and due to this feature, studies were started to reduce the RCS. The first efforts were devoted to radar absorption (RAM) materials. One particular type of RAM studied was called the analog absorber circuit, because it depended on the layers of the conductive material of the geometrical arrays, which acted as combinations of resistive, capacitive, and inductive circuit components, such as Salisbury screens and Jaumann absorbers [4].

Alternatively, it is proposed to use two cascading FSSs, which results in a configuration called an absorber/transmitter FSS. This proposal has been investigated by several researchers [2], [3], [5]. This structure is similar to the Salisbury screen; however, has the great advantage that it allows signals outside of the absorption band of interest to be transmitted through the structure.

To analyze microwave absorbers implemented with FSS there are different techniques in literature that are applied in several commercial electromagnetic problem analysis software. Those softwares use full wave analysis methods and generally require considerable computational effort. In addition, they have a limited number of optimization techniques [6] - [8]. 
The idea of this paper is to propose an absorber/transmitter FSS analysis technique that can be used in conjunction with any artificial intelligence technique that demands low computational effort. The proposed technique combines the equivalent circuit method [9], [10] with the scattering matrix technique [11], [12]. This work is an extension of [13], in which we improve the proposed modeling, analyze a second geometry, the square loop; construct two prototypes, and validate the proposed technique with experimental results.

\section{Proposed Structure}

The proposed structure is similar to the Salisbury screen, but differs from the classical structure in that it uses periodic structures in both the resistive layer and the metallic ground plane. It has a resistive screen, which is the first FSS away from the first FSS, comes the second FSS, at a distance of approximately $1 / 4$ wavelength. This second FSS is conductive and acts as a ground plane for the frequencies to be absorbed, allowing all other frequencies to pass through the structure with a minimum of attenuation. Fig. 1 illustrates the structure considered. The periodicities of the two FSS are identical, $p$. The dimensions of the resistive FSS are loop strip thickness, $w_{1}$, loop side length, $d_{1}$, dielectric thickness, $h_{1}$ and loop spacing, $g_{1}=p-d_{1}$. The conductive FSS dimensions are: loop strip thickness, $w_{2}$, loop side length, $d_{2}$, dielectric thickness, $h_{2}$ and loop spacing, $g_{2}=p-d_{2}$.

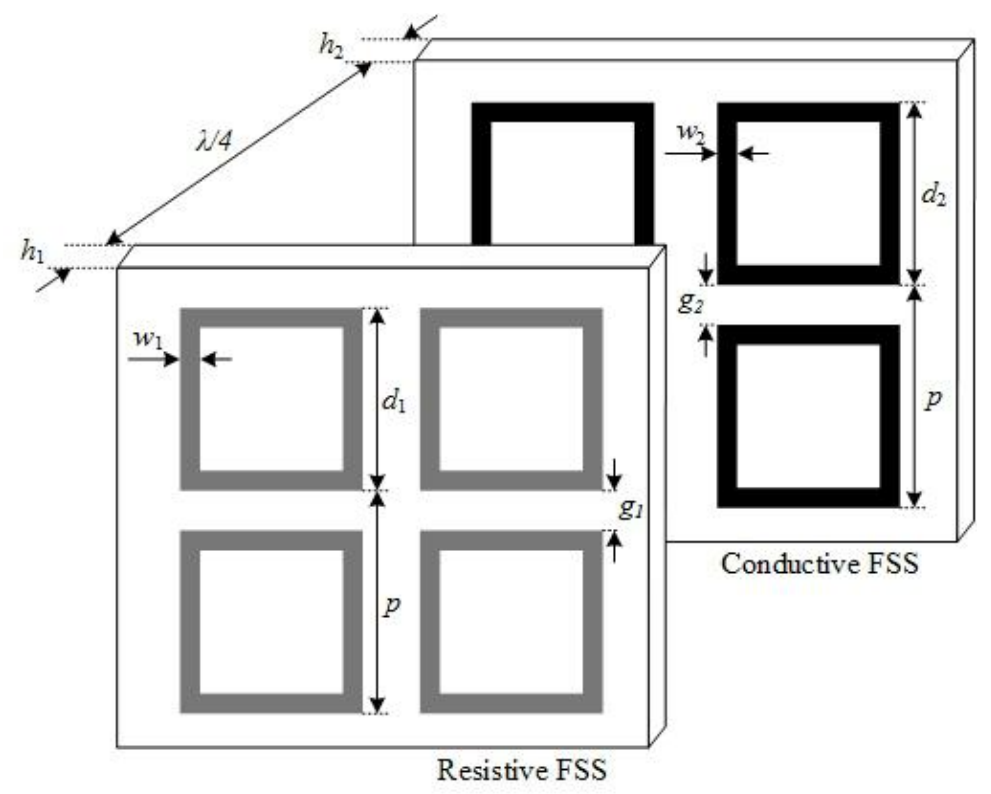

Fig. 1. Absorber/transmitter FSS.

The incident wave is considered a plane wave. The conductive FSS acts as a stopband filter, reflecting the desired frequency range and strongly attenuating these frequencies in the transmission direction. The resistive FSS, on the other hand, should strongly attenuate the reflected wave to the frequencies of interest, dissipating the electromagnetic energy in the resistive material cells. Thus, the composite structure functions as an absorber/transmitter FSS. Then, the absorption characteristics are achieved by placing a second FSS layer consisting of resistive square loops. A Nelco4000 substrate 
with thickness of $0.254 \mathrm{~mm}$ supported by a FR4 substrate was used and the surface resistance of the resistive square loops is chosen as $R_{S}=50 \Omega / \square$.

The main difference between the proposed structure and the Salisbury screen is that the latter reflects all frequencies outside the absorption frequency range, which can lead to a significant increase in multiple paths in the vicinity, while the FSS only attenuates the frequencies of interest, allowing the others to be transmitted through the structure with minimal attenuation.

The versatility of this structure has led to the interest in developing an analysis technique that can be used in combination with various computational intelligence and optimization techniques; and with low computational effort. A disadvantage of the technique is that the periodicities of the resistive and conductive structures must be identical.

\section{EQuivalent Circuit Method}

Initially, let's model the conductive FSS. Modeling for square loop geometry was developed in [14]. The equivalent circuit for square loop geometry is illustrated in Fig. 2 and corresponds to a series L-C circuit.

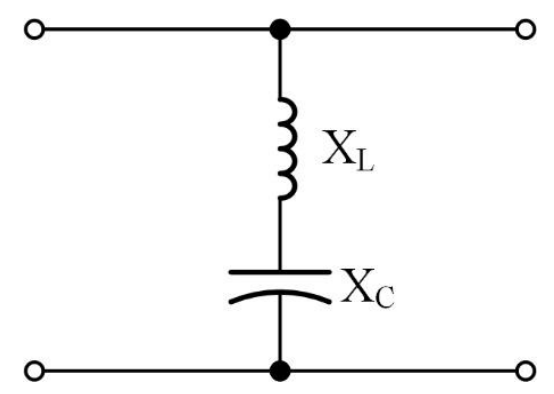

Fig. 2. Equivalent circuit for conductive FSS.

The normalized parameters of the square loop equivalent circuit, considering normal incidence, are:

$$
\begin{gathered}
\frac{\mathrm{X}_{\mathrm{L}}}{\mathrm{Z}_{0}}=F\left(p, w_{2}, \lambda, 0\right) \\
\frac{B_{C}}{Z_{0}}=4\left(\frac{d}{p}\right) \varepsilon_{\text {eef }} F\left(p, 2 w_{2}, \lambda, 0\right)
\end{gathered}
$$

where $Z_{0}$ is the air impedance and $\lambda$ is the wavelength.

The function $F(\bullet)$ was presented in [14] and $\varepsilon_{\text {eef }}$ is calculated as [15]:

$$
\varepsilon_{\text {eef }}=\varepsilon_{r}+\left(\varepsilon_{r}-1\right)\left(\frac{-1}{e^{10 N h_{2} / p}}\right)
$$

where $N$ is an index that depends on the geometry, the occupied area in each cell by the metal and is calculated according to the methodology adopted in [15].

The equivalent admittance for this circuit can be calculated as:

$$
Y=\frac{1}{X_{L}-1 / B_{C}}
$$


The transmission coefficient is calculated as:

$$
S_{21 C}=\frac{1}{\sqrt{1+0.25 Y^{2}}}
$$

Considering a perfectly conductive metal the reflection coefficient can be calculated as:

$$
S_{11 C}^{2}+S_{21 C}^{2}=1
$$

After this, we need to model the resistive structure. In general, a resistance (R) is added in the modeling of structures [16]. The equivalent circuit for square loop geometry is illustrated in Fig. 3, corresponding to a series R-L-C circuit.

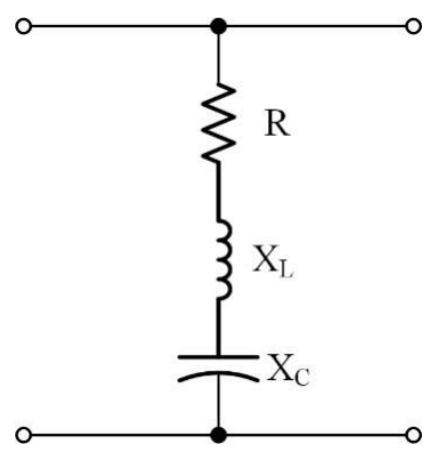

Fig. 3. Equivalent circuit for resistive FSS.

The normalized parameters of the square loop equivalent circuit, for a resistive FSS, are:

$$
\begin{gathered}
R=\left(\frac{S}{A}\right) R_{S} \\
\left(\frac{X_{C}}{Z_{0}}\right)^{-1}=\frac{4 p}{\lambda}\left[\ln \sec \psi+\frac{Q \operatorname{sen}^{4} \psi}{1+Q \cos ^{4} \psi}+\left(\frac{p}{4 \lambda}\right)^{2}\left(1-3 \cos ^{2} \psi\right)^{2} \operatorname{sen}^{4} \psi\right] \\
\left(\frac{X_{L}}{Z_{0}}\right)=\frac{p}{\lambda}\left[\ln \csc \psi+\frac{Q \cos ^{4} \psi}{1+Q \sin ^{4} \psi}+\left(\frac{p}{4 \lambda}\right)^{2}\left(1-3 \sin ^{2} \psi\right)^{2} \cos ^{4} \psi\right]
\end{gathered}
$$

where,

$$
\begin{gathered}
Q=\frac{1}{\sqrt{1-\left(\frac{p}{\lambda}\right)^{2}}}-1 \\
\psi=\frac{\Pi w}{2 p}
\end{gathered}
$$

where $R_{S}$ is the surface resistance of the material used as resistive surface, which in this work is 50 $\Omega / \Leftrightarrow, S=p^{2}$ and $A=2 w_{1}\left(d_{1}-w_{1}\right)$.

In this case, the total impedance of the structure is:

$$
Z=\left(R+j\left(X_{L}-X_{C}\right)\right)
$$

The reflection and transmission coefficients are calculated as [17]:

$$
S_{11 R}=\frac{-Z_{0}}{Z_{0}+2 Z}
$$




$$
S_{21 R}=\frac{2 Z}{Z_{0}+2 Z}
$$

\section{SCATtering Matrix TeChNiQue}

With the reflection and transmission coefficients of the resistive and conductive FSS, we can calculate these coefficients for the cascade structure to obtain absorption/transmission characteristics, as shown in Fig. 4. The interaction between the two FSS can be calculated using the scattering matrix technique since the distance between the two is large enough, i.e. the separation distance between the two FSS cannot be much shorter than the wavelength. Thus, the mutual coupling between the FSS can be neglected [18].

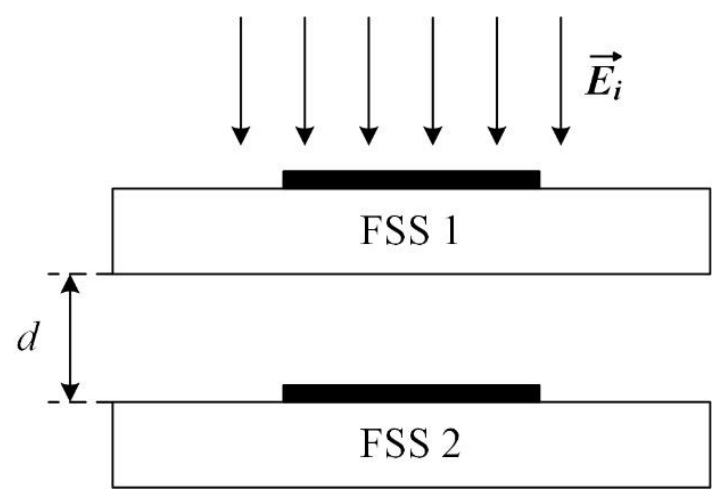

Fig. 4. Cascaded structures.

Using the scattering matrix technique [18], the final results for the transmission and reflection coefficients for the cascaded FSS are calculated as:

$$
\begin{gathered}
S_{21}=A-\left(\frac{B C}{D}\right) \\
S_{11}=-\left(\frac{C}{D}\right)
\end{gathered}
$$

where parameters $\mathrm{A}, \mathrm{B}, \mathrm{C}$ and $\mathrm{D}$ are calculated as:

$$
\left[\begin{array}{ll}
A & B \\
C & D
\end{array}\right]=\overline{\bar{S}}_{2} \overline{\bar{S}}_{1}
$$

and the scattering matrix of the nth structure is:

$$
\overline{\bar{S}}_{n}=\left[\begin{array}{cc}
S_{21 n}\left(1-\frac{S_{11 n}^{2}}{S_{21 n}}\right) & \frac{S_{11 n}}{S_{21 n}} e^{j 2 k l_{n}} \\
-\frac{S_{11 n}}{S_{21 n}} e^{-j 2 k l_{n}} & \frac{1}{S_{21 n}}
\end{array}\right]
$$

For the proposed structure, the reflection and transmission coefficients are calculated as:

$$
\begin{gathered}
S_{21}=\frac{S_{21 R} S_{21 C}}{1-S_{11 R} S_{11 C} e^{-j 2 k d}} \\
S_{11}=S_{11 R}+\frac{S_{21 C}^{2} S_{11 C}}{1-S_{11 R} S_{11 C} e^{-j 2 k d}} e^{-j 2 k d}
\end{gathered}
$$




\section{RESUlts AND DiscusSIONS}

In order to validate the proposed numerical technique two FSS square loop prototypes were constructed and the measured results obtained in [5] were used for the cross dipole case. For normal incidence measurement, the transmitter and receiver sides were connected to two identical ultrawideband horn antennas. The antennas have an average gain of $14 \mathrm{dBi}$. The distance from both antennas to the center of the structure was $1.30 \mathrm{~m}$. The frequency response of the prototypes was evaluated using a network vector analyzer (VNA) connected directly to both transmitting and receiving antennas. Four measurements were performed with the above-mentioned configuration. Unobstructed window measurements referred to as free space measurement (FS), and four with the FSS under test were performed. Fig. 5 illustrates the measurement setup. The equipment used was: two $700 \mathrm{MHz} 18 \mathrm{GHz}$ model SAS-571 horn antennas with wooden tripods, one Agilent Technologies model N5230A vector network analyzer, operating in the $300 \mathrm{kHz}$ to $13.5 \mathrm{GHz}$ range (with its due coaxial cables). In addition, one structure was used to support the absorber and has in one side RF absorbers and conductive foil. With this support, we reduce the noise caused by diffraction contamination.

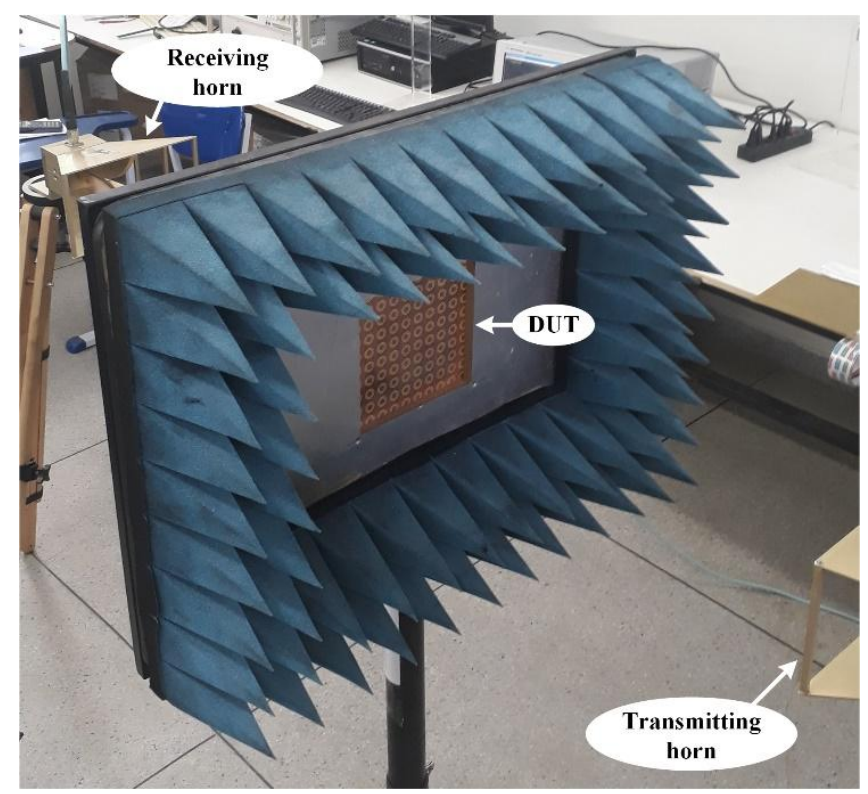

Fig. 5. Measurement setup.

The first prototype built has periodicity $p=42.5 \mathrm{~mm}$. The dielectric used in both FSS is the FR4, with dielectric constant $\varepsilon_{r}=4.4$. The resistive substrate has thickness $h_{1}=1.854 \mathrm{~mm}$ and the conductive substrate has thickness $h_{2}=1.6 \mathrm{~mm}$. The conductive FSS has dimensions: $d_{2}=31.95 \mathrm{~mm}$ and $w_{2}=2 \mathrm{~mm}$, while the resistive FSS has dimensions: $d_{1}=33.95 \mathrm{~mm}$ and $w_{1}=4 \mathrm{~mm}$. The distance between the FSS layers is $d=26.4 \mathrm{~mm}$. The two FSS were cascaded using Teflon spacers. Fig. 6 illustrates the built absorber. In Fig. 7 we can see the comparison between simulated and measured results for the S-parameters with normal incidence. Continuous line is the $S_{21}$ and dashed line is the $S_{11}$. The results show that the cascading structure suppresses 2 to $3 \mathrm{GHz}$ transmission. Our proposed 
technique shows better results in terms of resonance frequency and for the $S_{11}$ parameter than results obtained with HFSS, but for bandwidth, the results obtained with HFSS show better agreement. However, a good agreement between the results is observed.

The second built prototype has periodicity $p=24 \mathrm{~mm}$. The dielectric used in both FSS is the FR4, with dielectric constant $\varepsilon_{r}=4.4$. The resistive substrate has thickness $h_{1}=1.97 \mathrm{~mm}$ and the conductive substrate has thickness $h_{2}=1.54 \mathrm{~mm}$. The conductive FSS has dimensions: $d_{2}=21.5 \mathrm{~mm}$ and $w_{2}=2.2$ $\mathrm{mm}$, while the resistive FSS has dimensions: $d_{1}=21.5 \mathrm{~mm}$ and $w_{1}=3 \mathrm{~mm}$. There is a separation $d=$ $14 \mathrm{~mm}$, between the two layers. The two FSS were cascaded using an acrylic spacer. Figure 8 illustrates the constructed structure.

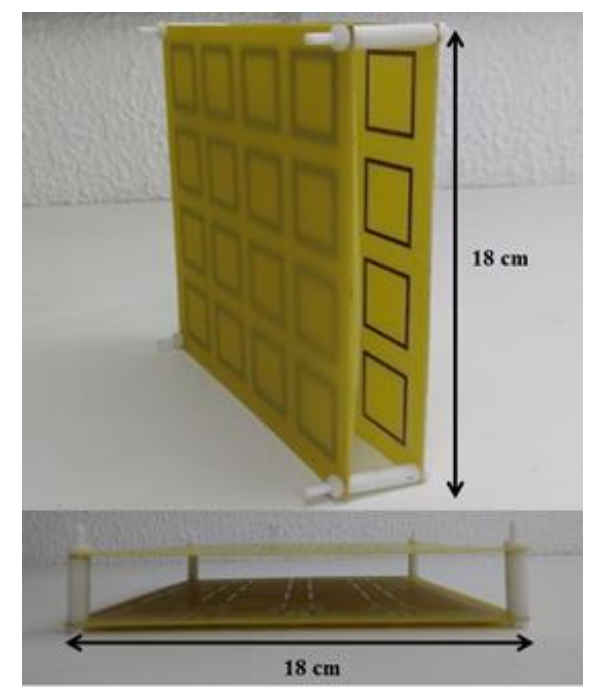

Fig. 6. First built prototype.

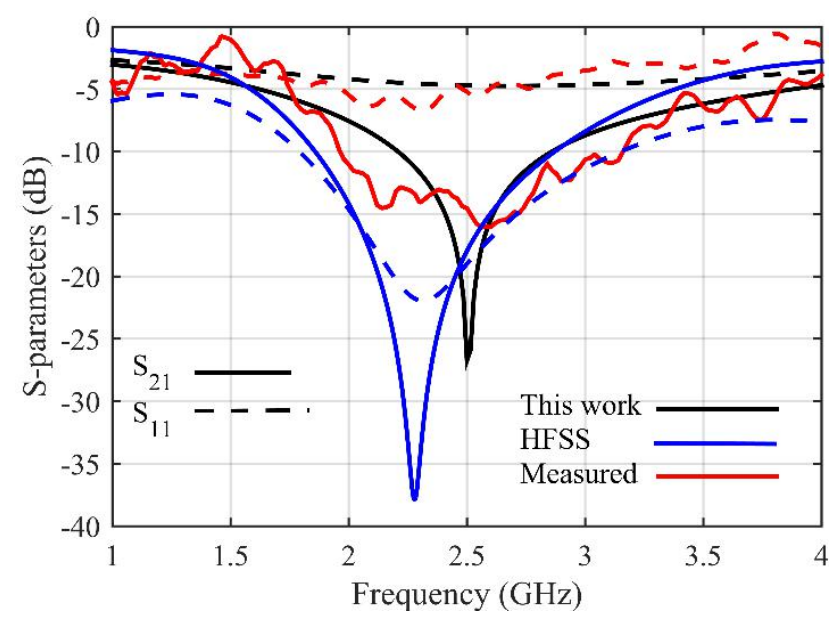

Fig. 7. Comparison between simulated and measured results for S-parameters of the first built prototype. 


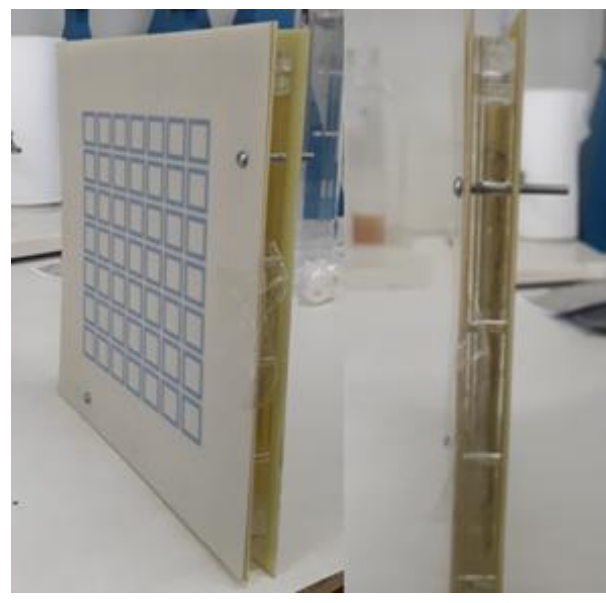

Fig. 8. Second built prototype.

In Figure 9 we can see the comparison between simulated and measured results, for the Sparameters. Again, our proposed technique shows better results in terms of resonance frequency and for the $S_{11}$ parameter than results obtained with HFSS, and again for bandwidth, the results obtained with HFSS show better agreement. However, a good agreement between the results is observed.

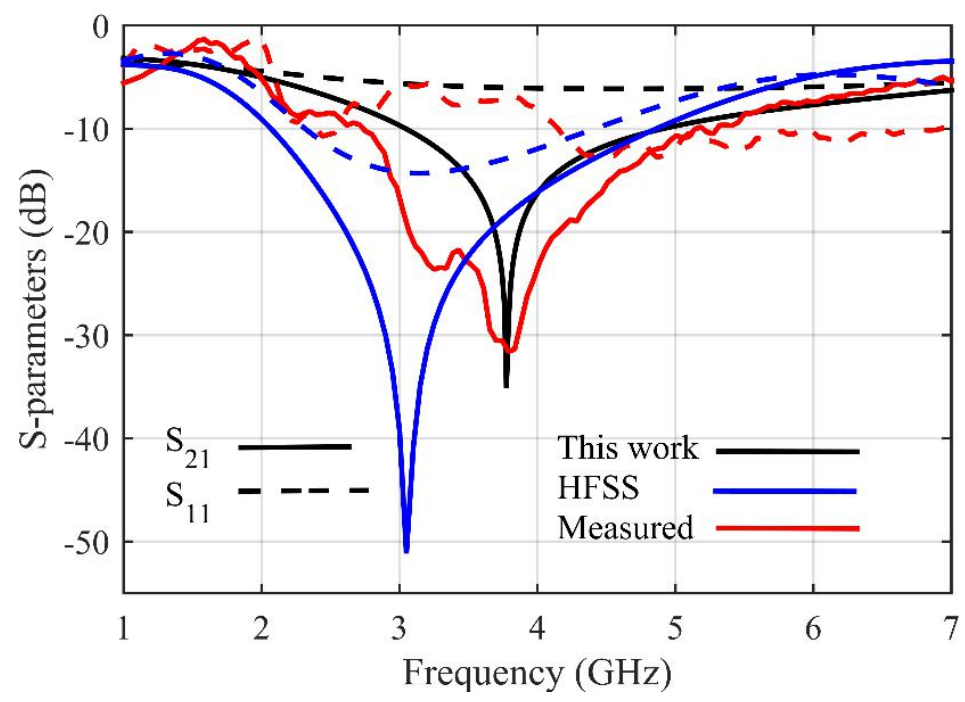

Fig. 9. Comparison between simulated and measured results for S-parameters of the second built prototype.

The third comparative case was obtained from the results measured in [5]. The considered periodicity is $p=28.57 \mathrm{~mm}$. The dielectric used in both FSS and FR4, with dielectric constant $\varepsilon_{r}=4.4$. The resistive FSS has thickness $h_{1}=0.8 \mathrm{~mm}$ and the conductive FSS has thickness $h_{2}=1.6 \mathrm{~mm}$. The conductive and resistive FSS square turns have identical dimensions: $d_{1}=d_{2}=20.58 \mathrm{~mm}$ and $w_{1}=w_{2}$ $=3.83 \mathrm{~mm}$. The gap between the two FSS is $d=10 \mathrm{~mm}$. In Fig, 10 we can see the comparison between the simulated and measured results for the S-parameters. Again, our proposed technique shows better results in terms of resonance frequency and for $S_{11}$ parameter than results obtained with HFSS, and again for bandwidth, the results obtained with HFSS show better agreement. However, a good agreement between the results is observed.

The results presented in Figures 7, 9 and 10 show a small divergence between the proposed model and that obtained by full wave simulation, for the three analyzed cases. As is well known, 3D 
numerical approach presents accurate results, but result in high computational costs. The equivalent circuit analysis, based on the approximation of the FSS as a lumped network, in contrast to the fullwave simulations, provides a good view of the physical properties and design of the periodic structure, but with less precise results in relation to the full- 3D wave. Thus, in our opinion, this may be the main contributor to the disagreement between the numerical results. The experimental results are also shown in the figures. It can be observed that in all cases, the measured results are closer to this work than those of full-wave ones. One of the factors that most influence the response of the structure is the distance between the layers, defined as $d$ in Figure 4. A small variation can cause a large displacement in the location of the absorption peak. This, together with the fact that the measurements were not carried out in a controlled environment, may be related to the fact of the displacement in relation to the results obtained by the HFSS.

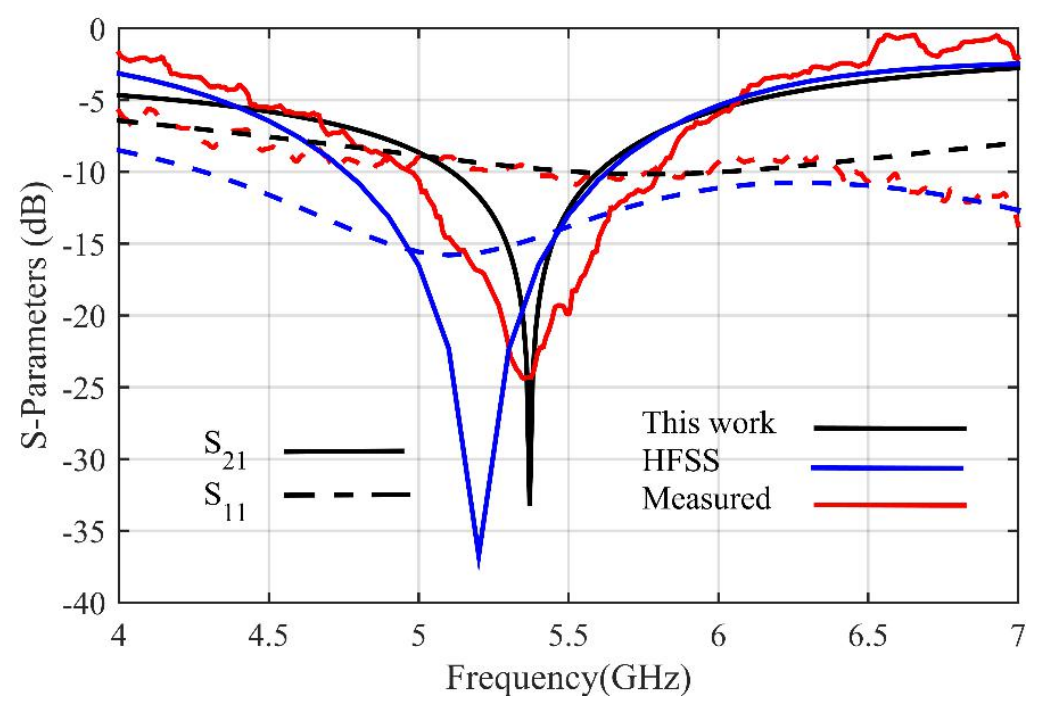

Fig. 10. Comparison between simulated and measured results for S-parameters of the built prototype in [5].

\section{CONCLUSIONS}

In this paper, an extension of [13] was proposed, investigating the use of a simple numerical technique for the analysis of absorber-transmitter FSS, with square loop and cross dipole geometries. The investigation was validated experimentally, with the construction of two square-loop prototypes and experimental results presented in [5]. The proposed technique allows a combination of optimization techniques and computational intelligence for absorbers synthesis with low computational effort. In addition, the work presented the modeling of resistive FSS, using the equivalent circuit method, which is not widespread in the literature. The simulated and measured results showed good agreement, which validates the proposed technique. About the HFSS results, a better convergence can be obtained with a test of convergence, to the refinement of the finite element mesh or the spectral resolution. 


\section{REFERENCES}

[1] V. B. Bregar, "Advantages of Ferromagnetic Nanoparticle Composites in Microwave Absorbers". IEEE Transactions on Magnetics, vol. 40, pp 1679 - 1684, May, 2004.

[2] A. Itou, O. Hashimoto, H. Yokokawa, and K. Sumi, "A Fundamental Study of a Thin $\lambda / 4$ Wave absorber Using FSS Technology”. Electronics and Communications in Japan (Part I: Communications), vol. 87 no.11, pp 77-86, May, 2004.

[3] A. Itou, H. Ebara, H. Nakajima, K. Wada, and O. Hashimoto, "An experimental study of a $\lambda / 4$ wave absorber using a frequency selective surface". Microwave and Optical Technology Letters, vol. 28, no. 5, pp 321-323, Jan, 2001.

[4] B. A. Munk, "Finite Antenna Arrays and FSS". Hoboken, NJ: Wiley-Interscience, 2003.

[5] G. I. Kiani, A. R. Weily, K. P. Esselle, "A novel absorb/transmit fss for secure indoor wireless networks with reduced multipath fading", IEEE Microwave and Wireless Components Letters, vol. 6, no.16, pp 378-380, May, 2006.

[6] COMSOL Multiphysiscs [On line]. July 23 2019. Available: https://br.comsol.com/.

[7] CST Studios [On line], July 23 2019, Available: https://www.3ds.com/products-services/simulia/products/cst-studiosuite/.

[8] ANSYS HFSS, [On line], July 23 2019, Available: https://www.ansys.com/products/electronics/ansys-hfss.

[9] A. L. P. S. Campos, A. M. Martins, and V. A. A. Filho, "Synthesis of frequency selective surfaces using genetic algorithm combined with the equivalent circuit method". Microwave and Optical Technology Letters, vol. 54, no. 8, pp 1893-1897, May, 2012.

[10] G. L. R. Araujo, A. L. P. S. Campos, and A. M. Martins, "Improvement of the Equivalent Circuit Method for Analysis of Frequency Selective Surfaces Using Genetic Algorithms and Rational Algebraic Models". Progress in Electromagnetics Research Letters, vol. 55, pp 67-74, Feb, 2015.

[11] T. L. Silva, A. L. P. S. Campos, A. G. d'Assunção, and R. H. C. Maniçoba, "A Comparative Study of Two Numerical Techniques to Analyze Double Screen Frequency Selective Surface". Microwave and Optical Technology Letters, vol. 55, pp 2206-2209, Jun, 2013.

[12] A. L. P. S. Campos, R. H. C. Maniçoba, L. M. Araujo, and A. G. d'Assunção, "Analysis of Simple FSS Cascading with Dual Band Response”. IEEE Transactions on Magnetics, vol. 46, pp 3345-3348, Jul, 2010.

[13] E. P. Santos and A. L. P. S. Campos, "A Simple Technique to Analysis of Microwave Absorbers using Frequency Selective Surfaces". In: SBMO/IEEE MTT-S International Microwave and Optoelectronics Conference - IMOC, Pernambuco, Brazil, 2015.

[14] R. J. Langley and E. A. Parker, "Equivalent circuit model for arrays of square loops". Electronics Letters, vol. 18, pp 294-296, Feb, 1982.

[15] F. Costa, A. Monorchio, and G. Manara, "Efficient Analysis of Frequency-Selective Surfaces by a Simple EquivalentCircuit Model". IEEE Antennas and Propagation Magazine, vol. 54, no. 4, pp 35 - 48, Sep, 2012.

[16] F. B. Gross and E. J. Kuster, "An Optimized Polarization Sensitive Salisbury Screen”. IEEE Transactions on Antennas and Propagation, vol. 35, no. 12, pp 1492 - 1495, Dec, 1987.

[17] R. Hall and R. Mittra, "Scattering from a periodic array of resistive strips". IEEE Transactions on Antennas and Propagation, vol. 33, no. 9, pp 1009-1011, Sep, 1985.

[18] S. W. Lee, G. Zarrillo, and C. L. Law, "Simple formulas for transmission through periodic metal grids or plates". IEEE Transactions on Antennas and Propagation, vol. 30, no. 5, pp 904-909, Sep, 1982. 